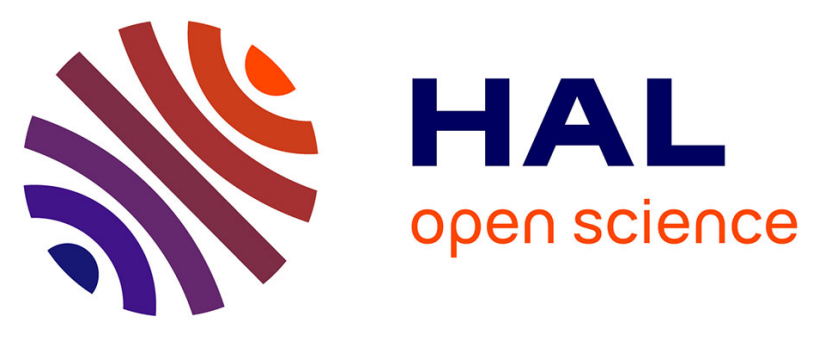

\title{
Laboratory X-ray characterization of a surface acoustic wave on GaAs: the critical role of instrumental convolution
}

Ludovic Largeau, Ibrahima Camara, Jean-Yves Duquesne, Catherine Gourdon, Pauline Rovillain, L. Thevenard, Bernard Croset

\section{To cite this version:}

Ludovic Largeau, Ibrahima Camara, Jean-Yves Duquesne, Catherine Gourdon, Pauline Rovillain, et al.. Laboratory X-ray characterization of a surface acoustic wave on GaAs: the critical role of instrumental convolution. Journal of Applied Crystallography, 2016, 31, pp. 2073. 10.1107/S1600576716015016 . hal-01363432v2

\section{HAL Id: hal-01363432 \\ https://hal.science/hal-01363432v2}

Submitted on 14 Dec 2016

HAL is a multi-disciplinary open access archive for the deposit and dissemination of scientific research documents, whether they are published or not. The documents may come from teaching and research institutions in France or abroad, or from public or private research centers.
L'archive ouverte pluridisciplinaire HAL, est destinée au dépôt et à la diffusion de documents scientifiques de niveau recherche, publiés ou non, émanant des établissements d'enseignement et de recherche français ou étrangers, des laboratoires publics ou privés. 


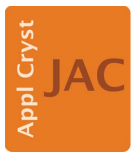

JOURNAL OF

APPLIED

CRYSTALLOGRAPHY

ISSN 1600-5767

Received 21 July 2016

Accepted 22 September 2016

Edited by G. Renaud, CEA-Grenoble DSM/ INAC/SP2M/NRS, Grenoble, France

Keywords: X-ray diffraction; surface acoustic waves; GaAs.

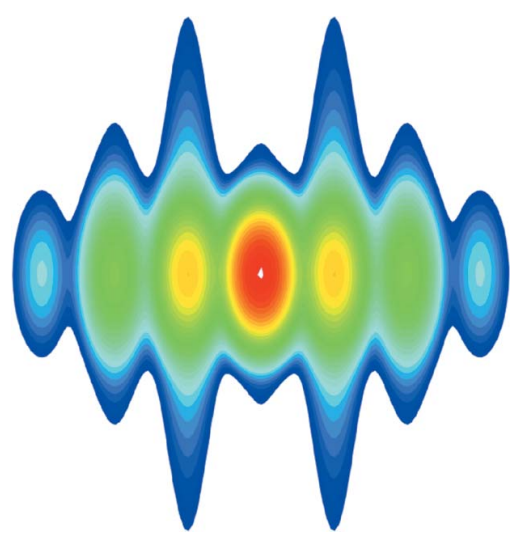

C 2016 International Union of Crystallography

\section{Laboratory X-ray characterization of a surface acoustic wave on GaAs: the critical role of instrumental convolution}

\author{
Ludovic Largeau, ${ }^{a}$ Ibrahima Camara, ${ }^{\text {b Jean-Yves Duquesne, }}{ }^{\text {b }}$ Catherine Gourdon, ${ }^{\text {b }}$ \\ Pauline Rovillain, ${ }^{b}$ Laura Thevenard ${ }^{b}$ and Bernard Croset $^{b *}$
}

${ }^{a}$ Centre de Nanosciences et de Nanotechnologies, CNRS, Université Paris-Sud, Université Paris-Saclay, 91460, Marcoussis, France, and ${ }^{\mathbf{b}}$ Sorbonne Universités, UPMC Université Paris 06, CNRS-UMR 7588, Institut des Nanosciences de Paris, 75005, Paris, France. *Correspondence e-mail: bernard.croset@insp.jussieu.fr

Surface acoustic waves of micrometre wavelength travelling on a monocrystal give diffraction satellites around each Bragg peak in an X-ray diffraction diagram. By using a four-crystal monochromator, a secondary two-crystal analyser and masks reducing the footprint to the part of the crystal containing the acoustic modulation, it is possible to observe these satellites on a GaAs (001) surface using a laboratory diffractometer. The finite extension of the satellite diffraction rods and of the crystal truncation rod perpendicular to the surface leads to geometrical correction factors when convoluted with the instrumental resolution function, which had previously been ignored. The calculation of these geometrical correction factors in the framework of the kinematic approximation allows the determination of the surface acoustic wave amplitude, and the study of its attenuation and its dependence on radiofrequency power and duty cycle. The ability to perform such determinations with a laboratory diffractometer should prove useful in optimizing surface acoustic waves, which are presently used in a broad range of condensed matter physics studies.

\section{Introduction}

Since the pioneering work of Hauer \& Burns (1975), a great deal of effort has been devoted to studying the interactions between acoustic waves and X-rays (see e.g. Entin et al., 1990; Roshchupkin \& Brunel, 1993; Tucoulou et al., 1997, 1998, 2001; Zolotoyabko \& Polikarpov, 1998; Sauer et al., 1999; Roshchupkin, Irzhak, Snigirev et al., 2011; Bojahr et al., 2012). One of the main motivations of these studies has been the tailoring of synchrotron light. For this reason and because of the high brilliance of synchrotron light, most of the recent studies have been performed using synchrotron facilities. Tucoulou et al. (2001) showed that a surface acoustic wave of some $10 \mu \mathrm{m}$ wavelength propagating on an $\mathrm{LiNbO}_{3}$ crystal leads to satellite diffraction peaks around each Bragg peak in a rocking curve. The $q$ separation between satellites being some thousandths of $\mathrm{nm}^{-1}$, the very close proximity of the satellites to the Bragg peak implies the use of both a high-resolution diffractometer and a good-quality monocrystal. While these authors analysed their results in the framework of the kinematic theory of diffraction, some subsequent work has been analysed using the dynamic theory (Schelokov et al., 2004; Tucoulou et al., 2005).

In the meantime, interest in surface acoustic waves (SAWs) has grown. Excited electrically using interdigitated transducers (IDTs), they are routinely used as band-pass filters in radiofrequency (RF) microelectronics up to $3 \mathrm{GHz}$. In more 
academic environments, they have recently emerged as an efficient tool for carrier and spin control in semiconductor and metallic nanostructures, with applications foreseen in quantum information technology (Sanada et al., 2011; Hermelin et al., 2011). Their versatility and the wide possibilities of tailoring their interaction with electronic or magnetic excitations by using the toolbox of wave mechanics (interference, focusing, wavefront and pulse shaping etc.) make SAWs appealing to a growing number of condensed matter physicists (Li et al., 2014; Schülein et al., 2015; Thevenard et al., 2016).

Because of this active development of SAW studies, there is a real need for an easy and versatile method to quantitatively measure their amplitude in view of optimizing the design of IDTs. This has been done in the past using optical light diffraction or interferometry (Lean \& Powell, 1972; Royer \& Dieulesaint, 2001; de Lima et al., 2003; Singh \& Adenwalla, 2015). Recently, SAW studies have been performed on a laboratory diffractometer without explicit measurements of the acoustic amplitude (Roshchupkin, Irzhak, Plotitsyna et al., 2011; Insepov et al., 2015). We show here on a pristine GaAs substrate that quantitative measurements of the acoustic amplitude can be performed using X-rays on a laboratory diffractometer through a careful experimental procedure and an accurate data analysis.

\section{Experimental setup and procedure}

\subsection{Experimental setup}

High-resolution X-ray diffraction measurements were carried out on a PANalytical X'pert Pro diffractometer equipped with a sealed $\mathrm{Cu}$ tube and in a triple-axis geometry. A primary four-crystal $\mathrm{Ge}(220)$ monochromator and a secondary two-crystal $\mathrm{Ge}(220)$ analyser were used. Such a geometry allows one to select $\mathrm{Cu} K \alpha_{1}$ radiation of X-ray wavelength $\lambda_{\mathrm{RX}}=0.1540562 \mathrm{~nm}$.

Using the piezoelectric properties of GaAs, we deposited two IDTs parallel to the $\langle 110\rangle$ direction of an epiready

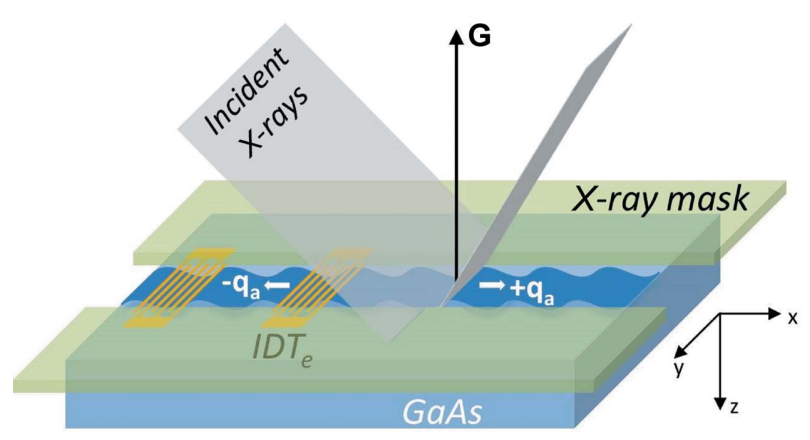

Figure 1

Schematic of the experiment (not to scale): the central metallic transducer $\left(\mathrm{IDT}_{\mathrm{e}}\right)$ deposited directly on GaAs emits bidirectionally a surface acoustic wave. The incident X-ray beam probes the induced displacements at different distances $x=0-7 \mathrm{~mm}$. The glass masks ensure the X-rays are only scattered off the perturbed region of GaAs. substrate. The first IDT, IDT $_{\mathrm{e}}$, allows the excitation of an acoustic wave propagating perpendicularly to this direction. The second IDT was deposited $l_{\text {gap }}=3 \mathrm{~mm}$ to its left (Fig. 1). This is the standard SAW filter configuration which allows electrical monitoring of the transmitted SAW shape (Royer \& Dieulesaint, 2001). In this work, we have explored instead the right side of the $\mathrm{IDT}_{\mathrm{e}}$ using X-rays. The period of the IDT fingers was nominally $\lambda_{a}=5 \mu \mathrm{m}$, the metallization ratio 0.5 , the aperture $W=2 \mathrm{~mm}$ and the number of IDT finger pairs $N=$ 60. The electrodes were made of $\mathrm{Cr} / \mathrm{Au}$ of nominal thickness 10/100 $\mathrm{nm}$. The excitation signal was brought to the IDTs by means of coaxial cables and coplanar waveguides with straight round bond-wires. Unless specified, the RF power was applied continuously (CW SAW mode). Otherwise it was modulated at low frequency by a square wave of period $T_{\bmod }=20 \mu \mathrm{s}$ (Mod SAW mode). The resonance frequency of the $\mathrm{IDT}_{\mathrm{e}}$ was measured to be $548 \pm 3 \mathrm{MHz}$.

Operando measurement of the acoustic wave propagation was achieved by collecting rocking curves and reciprocalspace maps. The crossed slits did not allow us to define a sharp $\mathrm{X}$-ray probe. Therefore, the use of masks for X-rays was required to limit the diffraction to the part of the crystal containing the acoustic modulation. We placed glass masks with an $s_{y}=2 \mathrm{~mm}$ width gap just above the surface but not touching it in order not to disturb the acoustic wave propagation. The spot size in the direction of the SAW propagation was $s_{x}=1.8 \mathrm{~mm}$ for the (002) reflection and $s_{x}=0.9 \mathrm{~mm}$ for the (004) reflection. The centre of the spot was positioned at a distance ranging from $x=0$ to $7 \mathrm{~mm}$ to the right of the $\mathrm{IDT}_{\mathrm{e}}$ (Fig. 1). Great care was taken to avoid any strain when attaching the sample to the holder.

\subsection{Experimental procedure}

The X-ray spot was first positioned at $x=2.1 \mathrm{~mm}$ from the $\mathrm{IDT}_{\mathrm{e}}$. The latter was excited at its resonance frequency with RF incoming powers ranging from $P_{\text {elec }}=40 \mathrm{~mW}$ to $P_{\text {elec }}=$ $320 \mathrm{~mW}$. Figs. 2, 3, 6 and 7 show rocking curves around the (002) and (004) Bragg peaks of the GaAs substrate. At the $\mathrm{IDT}_{\mathrm{e}}$ resonance, the propagation of the SAW induces a sinusoidal modulation of the crystal in the propagation direction. Therefore, around each Bragg peak of position $\mathbf{Q}_{\mathrm{B}}$ we expect diffraction satellites at positions given by $\mathbf{Q}=\mathbf{Q}_{\mathrm{B}}+p \mathbf{q}_{\mathrm{a}}$, where $p$ is the satellite order, $q_{\mathrm{a}}=2 \pi / \lambda_{\mathrm{a}}=1.26 \times 10^{-3} \mathrm{~nm}^{-1}$ is the acoustic wavevector and $\lambda_{\mathrm{a}}=5 \mu \mathrm{m}$. The in-plane and the normal components of $\mathbf{Q}$ are given by $Q_{/ /}=2 \pi /$ $\lambda_{\mathrm{RX}}[\cos (2 \theta-\omega)-\cos (\omega)]$ and $Q_{z}=-2 \pi / \lambda_{\mathrm{RX}}[\sin (2 \theta-\omega)+$ $\sin (\omega)]$, respectively. The angular positions of the satellites, $\omega_{p}$ and $2 \theta_{p}$, are then given by $1 / \lambda_{\mathrm{RX}}\left[\cos \left(2 \theta_{p}-\omega_{p}\right)-\cos \left(\omega_{p}\right)-\right.$ $\left.\cos \left(2 \theta_{\mathrm{B}}-\omega_{\mathrm{B}}\right)+\cos \left(\omega_{\mathrm{B}}\right)\right]=p / \lambda_{\mathrm{a}}$ and $1 / \lambda_{\mathrm{RX}}\left[\sin \left(2 \theta_{p}-\omega_{p}\right)+\right.$ $\left.\sin \left(\omega_{p}\right)-\sin \left(2 \theta_{\mathrm{B}}-\omega_{\mathrm{B}}\right)-\sin \left(\omega_{\mathrm{B}}\right)\right]=0$, respectively. Since the satellites appear for low values of $\delta \omega=\omega-\omega_{\mathrm{B}}$ of some hundredths of a degree, the components of $\mathbf{Q}$ for symmetric reflections are simplified as $Q_{/ /}=Q_{\mathrm{B}} / 2[\delta(2 \theta-\omega)-\delta(\omega)]$ and $Q_{z}=Q_{\mathrm{B}}\{1+\cot (\theta) / 2[\delta(2 \theta-\omega)+\delta(\omega)]\}$. Therefore, the rocking curves are equivalent to $Q_{/ /}$scans and $(\omega / 2 \theta, \omega)$ intensity maps are equivalent to $\left(Q_{/ /}, \delta Q_{z}\right)$ intensity maps. A 
rapid analysis of the (002) $Q_{/ /}$scans (Fig. 2) shows that, as expected, the satellite features are symmetric around the Bragg position, periodic with a period almost equal to the nominal acoustic wavevector, and that their intensities increase with the incoming RF power. The conclusions are less clear for the (004) $Q_{/ /}$scans (Fig. 3) since the satellite features are not so well separated, but once again the diffuse intensities outside the Bragg condition increase with the incoming RF power. In order to carefully check that no thermal drift appears on the diffraction patterns when applying the electrical power on the IDT, we also excited it off its resonance, at $570 \mathrm{MHz}$. Diffraction patterns identical to the one without any $\mathrm{RF}$ excitation were then recovered.

Fig. 4(a) shows an $(\omega / 2 \theta, \omega)$ intensity map around the (002) Bragg peak in the presence of the acoustic wave. Satellite rods parallel to the $Q_{z}$ direction clearly appear. Note that the extension of these rods in the $Q_{z}$ direction varies with the satellite order. In the next two sections, we will describe how to perform a quantitative analysis of these experimental features in order to extract the acoustic wave amplitude and to study its physical behaviour as a function of different parameters: incoming RF power, distance to the $\mathrm{IDT}_{\mathrm{e}}$ and $\mathrm{SAW}$ duty cycle (ratio of the 'on' time to the modulation period).

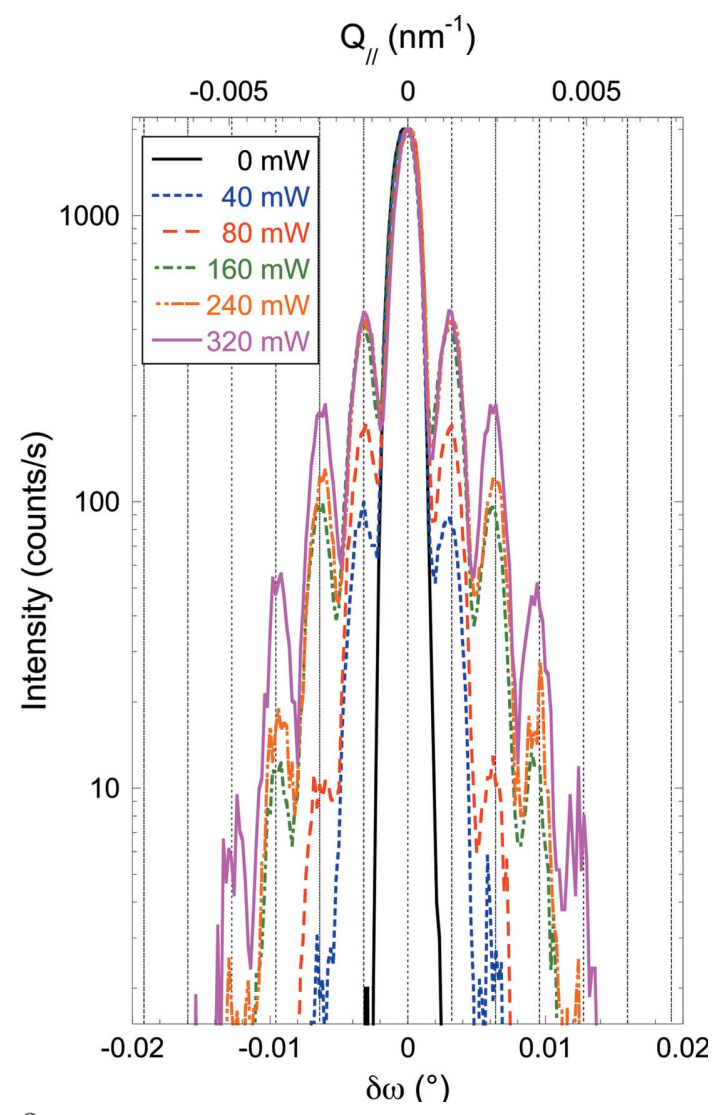

Figure 2

Rocking curves around the (002) Bragg peak taken $2.1 \mathrm{~mm}$ from the $\mathrm{IDT}_{\mathrm{e}}$ for increasing incoming RF power. The vertical lines are a guide for the eyes at $Q_{/ /}= \pm p q_{a}$. In order to show clearly the satellite evolution, all the intensity maxima have been normalized to the maximum value at $P=$ 0 .

\section{Modelling and data reduction}

In order to obtain a good estimate of the X-ray diffracted intensity, the first step is to get an accurate description of the atomic displacements associated with the SAW travelling in the crystal. Note that, for a wavelength of the order of $5 \mu \mathrm{m}$, harmonic displacements of the order of $0.05 \mathrm{~nm}$ lead to a strain of the order of $6 \times 10^{-5}$. For such a small strain, the acoustic branch of the phonons can be calculated in the framework of linear elasticity of continuous media. In the case of cubic symmetry, for a (001) surface and propagation in the $\langle 110\rangle$ direction, the surface acoustic mode is a Rayleigh mode and one obtains for atomic displacements (Royer \& Dieulesaint, 2001)

$$
\begin{aligned}
& \mathbf{u}(x, y, z) \\
& =\left(\begin{array}{l}
u_{x} \\
u_{y} \\
u_{z}
\end{array}\right)=\left[\begin{array}{c}
U_{x}(z) \cos \left(q_{\mathrm{a}} x-\omega_{\mathrm{a}} t\right) \\
0 \\
U_{z}(z) \sin \left(q_{\mathrm{a}} x-\omega_{\mathrm{a}} t\right)
\end{array}\right] \\
& =\left[\begin{array}{c}
2 U \exp \left(-p_{\mathrm{r}} q_{\mathrm{a}} z\right) \cos \left(p_{\mathrm{i}} q_{\mathrm{a}} z+\varphi / 2\right) \cos \left(q_{\mathrm{a}} x-\omega_{\mathrm{a}} t\right) \\
0 \\
2 r U \exp \left(-p_{\mathrm{r}} q_{\mathrm{a}} z\right) \sin \left(p_{\mathrm{i}} q_{\mathrm{a}} z+\varphi / 2-\psi\right) \sin \left(q_{\mathrm{a}} x-\omega_{\mathrm{a}} t\right)
\end{array}\right],
\end{aligned}
$$

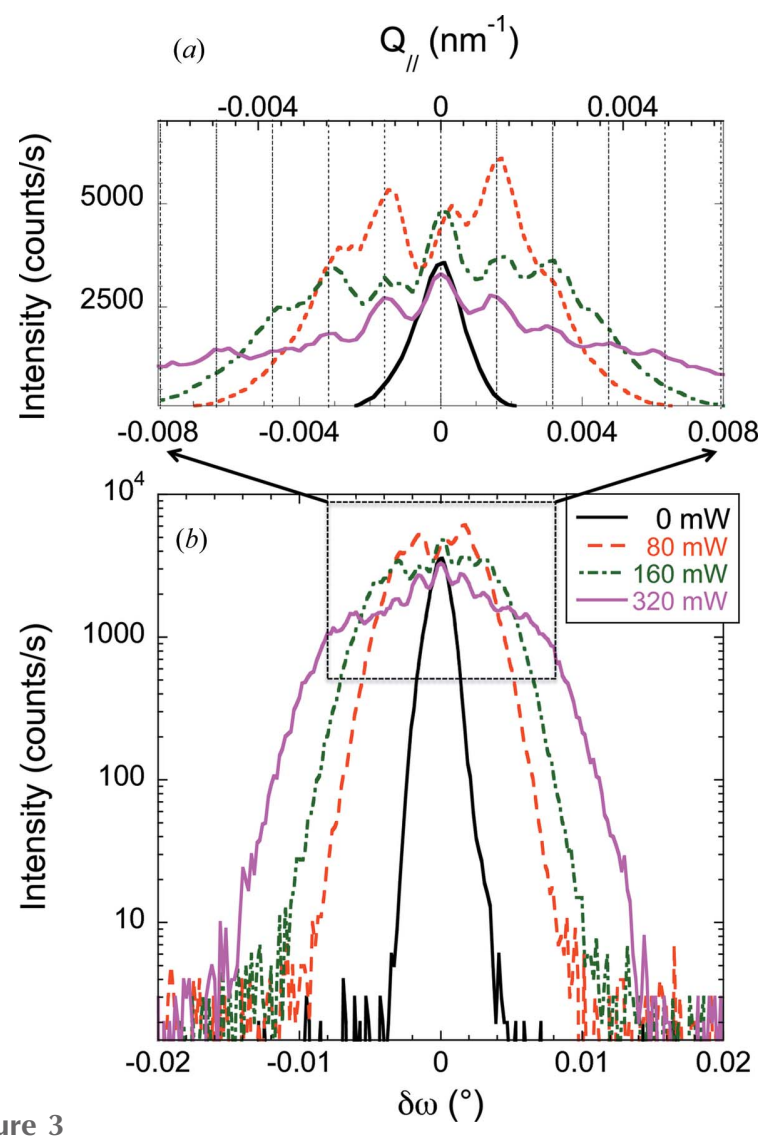

Figure 3

Rocking curves around the (004) Bragg peak taken $2.1 \mathrm{~mm}$ from the $\mathrm{IDT}_{\mathrm{e}}$ for increasing incoming RF power. (a) Intensity in linear scale. The vertical lines are a guide for the eyes at $Q_{/ /}= \pm p q_{a}$. (b) Intensity in logarithmic scale. 
where $q_{\mathrm{a}}=2 \pi / \lambda_{\mathrm{a}}$ and $\omega_{\mathrm{a}}$ are the wavevector modulus and the angular frequency of the SAW, respectively, and $r, p_{\mathrm{r}}, p_{\mathrm{i}}, \varphi, \psi$ are coefficients which depend on the elastic constants but are independent of the wave amplitude $U$. For GaAs using $C_{11}=$ $118.4 \mathrm{GPa}, C_{12}=53.7 \mathrm{GPa}, C_{44}=59.1 \mathrm{GPa}$ (Cottam \& Saunders, 1973), one obtains $r=1.34, p_{\mathrm{r}}=0.5, p_{\mathrm{i}}=-0.48, \varphi=-2.10$, $\psi=2.61$, so that the surface atomic $x$ and $z$ displacement amplitudes at $z=0$ are simply obtained as a function of $U$ by $u_{x}(z=0)=0.99 U$ and $u_{z}(z=0)=-1.33 U$. It is worth noting that (i) the expression of $\mathbf{u}$ differs noticeably from a simple exponential damping used by previous authors (Tucoulou $e t$ al., 2001) and (ii) a calculation performed in the framework of elasticity of continuous media implicitly assumes that all the atoms of the crystallographic unit cell have the same displacement.

Since the sound velocity, $v_{\mathrm{a}}=\omega_{\mathrm{a}} / q_{\mathrm{a}}$, is much lower than the light velocity, the acoustic strain can be considered as quasistatic (Tucoulou et al., 2001). Using the description of the atomic displacements, we can evaluate the amplitude $A(\mathbf{Q})$ of the diffracted X-ray wave in the framework of a kinematic approximation. The principle of the calculation was developed by Tucoulou et al. (2001), on the basis of earlier work of Entin et al. (1990). For a semi-infinite crystal $A(\mathbf{Q})$ can be written

$$
\begin{aligned}
A(\mathbf{Q}) \propto & F(\mathbf{Q}) \sum_{n_{x}=-\infty}^{\infty} \sum_{n_{y}=-\infty}^{\infty} \sum_{n_{z}=0}^{\infty}\left[\exp \left(-\tilde{\mu} R_{z}\right) \exp (i \mathbf{Q} \cdot \mathbf{R})\right] \\
\propto & F(\mathbf{Q}) \sum_{n_{x}=-\infty}^{\infty} \sum_{n_{y}=-\infty}^{\infty} \sum_{n_{z}=0}^{\infty}\left\{\exp \left(-\widetilde{\mu} R_{z}\right) \exp \left[i \mathbf{Q} \cdot\left(\mathbf{R}_{0}+\mathbf{u}\right)\right]\right\} \\
\propto & F(\mathbf{Q}) \sum_{G_{y}} \delta\left(Q_{y}-G_{y}\right) \sum_{n_{x}=-\infty}^{\infty} \sum_{n_{z}=0}^{\infty}\left(\exp \left(-\tilde{\mu} n_{z} a_{z}\right)\right. \\
& \times \exp \left[i\left(Q_{x} n_{x} a_{x}+Q_{z} n_{z} a_{z}\right)\right] \\
& \times \exp \left\{i\left[Q_{x} U_{x}\left(n_{z} a_{z}\right) \cos \left(q_{\mathrm{a}} n_{x} a_{x}\right)\right]\right\} \\
& \left.\times \exp \left\{i\left[Q_{z} U_{z}\left(n_{z} a_{z}\right) \sin \left(q_{\mathrm{a}} n_{x} a_{x}\right)\right]\right\}\right)
\end{aligned}
$$

where $F(\mathbf{Q})$ is the structure factor of the elementary mesh, $\mathbf{R}$ is the position of the $\left(n_{x}, n_{y}, n_{z}\right)$ crystal node, $\mathbf{R}_{0}$ is its position in the absence of the SAW and $a_{x}, a_{y}, a_{z}$ are the elementary vectors of the direct lattice. $\delta$ is the Dirac distribution, $G_{y}$ is the $y$ component of a reciprocal-lattice node $\mathbf{G}$ and $\tilde{\mu}=$ $(1 / 2) \mu_{\mathrm{GaAs}}[1 / \sin (\omega)+1 / \sin (2 \theta-\omega)]$ is the effective absorption coefficient for the X-ray amplitude. The $1 / 2$ factor accounts for the fact that $\mu_{\mathrm{GaAs}}$, the standard absorption coefficient, is defined with respect to the diffracted X-ray intensity (not amplitude), the importance of which will become apparent in the following since the satellite intensities are controlled by a subtle balance between X-ray absorption and acoustic wave amplitude decrease.

Defining $\rho_{\mathbf{Q}}\left(n_{z} a_{z}\right)$ and $\zeta_{\mathbf{Q}}\left(n_{z} a_{z}\right)$ by $\rho_{\mathbf{Q}} \sin \left(\zeta_{\mathbf{Q}}\right)=U_{x} Q_{x}$ and $\rho_{\mathbf{Q}} \cos \left(\zeta_{\mathbf{Q}}\right)=U_{z} Q_{z}, A(\mathbf{Q})$ yields

$$
\begin{aligned}
A(\mathbf{Q}) \propto & F(\mathbf{Q}) \sum_{G_{y}} \delta\left(Q_{y}-G_{y}\right) \sum_{n_{x}=-\infty}^{\infty} \sum_{\infty}\left[\exp \left(-\tilde{\mu} n_{z} a_{z}\right)\right. \\
& \times \exp \left[i\left(Q_{x} n_{x} a_{x}+Q_{z} n_{z} a_{z}\right)\right] \\
& \left.\times \exp \left(i\left\{\rho_{\mathbf{Q}}\left(n_{z} a_{z}\right) \sin \left[q_{a} n_{x} a_{x}+\zeta_{\mathbf{Q}}\left(n_{z} a_{z}\right)\right]\right\}\right)\right] .
\end{aligned}
$$

Using the identity $\exp (i t \sin \theta)=\sum_{p=-\infty}^{\infty} J_{p}(t) \exp (i p \theta)$, where $J_{p}$ is the Bessel function of the first kind of integer order $p$, $A(\mathbf{Q})$ can be written

$$
\begin{aligned}
A(\mathbf{Q}) \propto & F(\mathbf{Q}) \sum_{\mathbf{G}_{/ /}} \sum_{p=-\infty}^{\infty} \delta\left(\mathbf{Q}_{/ /}+p \mathbf{q}_{\mathrm{a}}-\mathbf{G}_{/ /}\right) \\
\times & \sum_{n_{z}=0}^{\infty} \exp \left(i Q_{z} n_{z} a_{z}\right) \exp \left(-\widetilde{\mu} n_{z} a_{z}\right) \\
& \times J_{p}\left[\rho_{\mathbf{Q}}\left(n_{z} a_{z}\right)\right] \exp \left[i \zeta_{\mathbf{Q}}\left(n_{z} a_{z}\right)\right] .
\end{aligned}
$$

Since we have $q_{\mathrm{a}} a_{z} \ll 1$, the variation with $n_{z}$ of $\rho_{\mathbf{Q}}$ and $\zeta_{\mathbf{Q}}$ is slow. Similarly, we have $\tilde{\mu} a_{z} \ll 1$ and the variation with $n_{z}$ of $\exp \left(-\tilde{\mu} n_{z} a_{z}\right)$ is slow. Therefore, for each rod, satellite rod or crystal truncation rod, $A(\mathbf{Q})$ has strong maxima around each Bragg value $G_{z}$ of $Q_{z}$. For the calculation of the profiles of these maxima, the summation on $n_{z}$ can be replaced by an integral, leading to

$$
A(\mathbf{Q}) \propto F(\mathbf{Q}) \sum_{\mathbf{G}} \sum_{p=-\infty}^{\infty} \delta\left(\mathbf{Q}_{/ /}+p \mathbf{q}_{\mathrm{a}}-\mathbf{G}_{/ /}\right) H_{p, \mathbf{G}}\left(Q_{z}-G_{z}\right)
$$

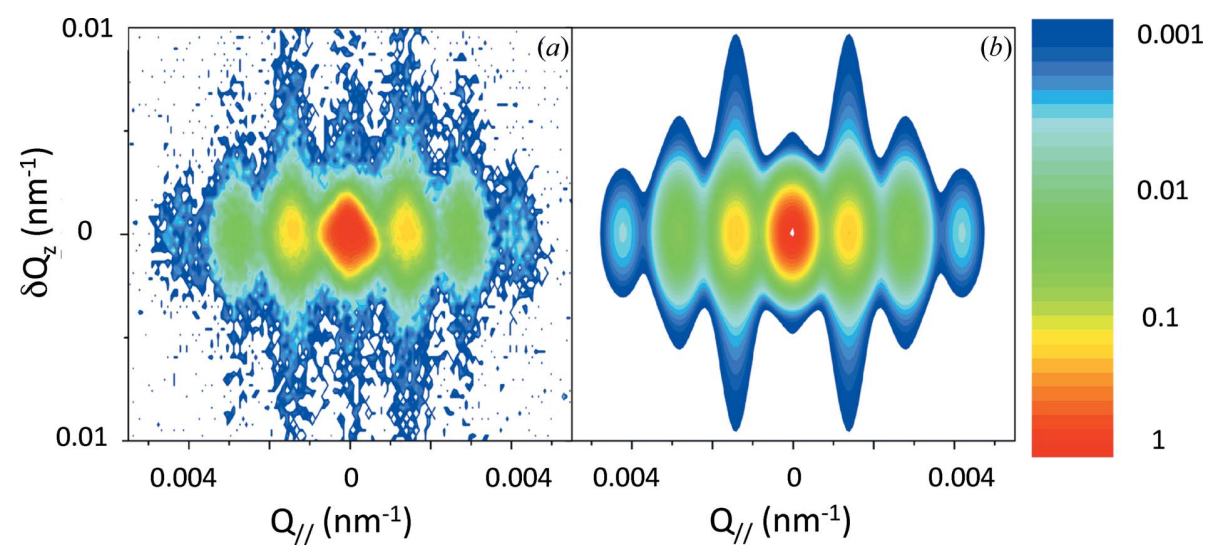

Figure 4

Measured $(a)$ and calculated $(b)(\omega / 2 \theta, \omega)$ intensity maps around the $(002)$ Bragg peak $\left(x=2.1 \mathrm{~mm}, P_{\text {elec }}=160 \mathrm{~mW}\right)$. Note that, as the colour scale is logarithmic, the $q_{z}$ width of the fundamental peak is much lower than that of the satellite peaks. 


$$
H_{p, \mathbf{G}}\left(q_{z}\right)=\int_{0}^{\infty} \exp \left(i q_{z} z\right) \exp (-\tilde{\mu} z) J_{p}\left[\rho_{\mathbf{G}}(z)\right] \exp \left[i \zeta_{\mathbf{G}}(z)\right] \mathrm{d} z
$$

This expression shows that every Bragg peak is decorated by satellites in the $q_{x}$ direction at positions $p \mathbf{q}_{a}$. While Bragg peaks and satellites have a $\delta$ profile in the $\mathbf{Q}_{/ /}=\left(Q_{x}, Q_{y}\right)$ plane, their extension and their profile in the $Q_{z}$ direction are controlled both by the finite penetration of the X-rays due to absorption and by the finite extension of the Rayleigh mode in the $z$ direction. For the sake of simplicity, we will consider, in the following, the case of symmetric reflections for which $Q_{x}=0$, but the conclusions are valid in the general case. For $Q_{x}=0$, we have $\zeta=0$ and $\rho=2 r U Q \exp \left(-p_{\mathrm{r}} q_{\mathrm{a}} z\right) \times$ $\sin \left(p_{\mathrm{i}} q_{\mathrm{a}} z+\varphi / 2-\psi\right)$. Since near $t=0$ we have $J_{0}(t)=1+$ $O\left(t^{2}\right)$ and $J_{p}(t) \simeq t^{p} / 2^{p} p$ !, the integrand factor in $H$ behaves like $\exp \left[-\left(\widetilde{\mu}+p p_{\mathrm{r}} q_{\mathrm{a}}\right) z\right]=\exp \left[-\left(\widetilde{\mu}+p \mu_{\mathrm{a}}\right) z\right]$ for large $z$ with $\mu_{\mathrm{a}}=0.63 \mu \mathrm{m}^{-1}$. From the NIST Standard Reference Database 126 (http://www.nist.gov/pml/data/xraycoef/) we have $\mu_{\text {GaAs }}=$ $0.039 \mu \mathrm{m}^{-1}$, leading to $\tilde{\mu}_{002}=0.14 \mu \mathrm{m}^{-1}$ and $\tilde{\mu}_{004}=$ $0.067 \mu \mathrm{m}^{-1}$. For small $U Q$, the asymptotic behaviour of the Bessel functions is reached for small values of $z$ and the $q_{z}$ profile of the diffracted intensity is essentially Lorentzian with a width $(1 / 2)\left(\tilde{\mu}+p \mu_{a}\right)$. The width of the $q_{z}$ profiles increases with $p$, the Bragg peak being significantly narrower particularly in the (004) case. For larger values of $U Q$, the oscillations of the Bessel function must be taken into account to precisely describe the $q_{z}$ profiles but the main behaviour is kept as can be seen in the intensity map of Fig. 4: the $q_{z}$ width increases with the satellite order, the Bragg peak $q_{z}$ width being notably narrower than that of the satellite peaks.

So far, we have calculated, like others before us (Tucoulou et al., 2001), the diffracted amplitude $A(\mathbf{Q})$ and the 'natural' intensity $I_{\text {nat }}=A A^{*}$ in the $q_{x}, q_{y}, q_{z}$ space. To obtain the effective intensity, $I_{\text {eff }}$, one must convolute the natural intensity with the 'instrumental' resolution function. This function accounts for several sources of peak broadening: angular resolutions, monochromaticity of the X-ray beam and finitesize effects (footprint size, crystal defects etc.). The small ratio between $q_{\mathrm{a}}$ and $Q, q_{\mathrm{a}} / Q_{002}=55 \times 10^{-6}$ and $q_{\mathrm{a}} / Q_{004}=$ $27.6 \times 10^{-6}$, has two consequences: first, the resolution function can be taken as constant when exploring the intensity landscape around each Bragg peak; second, as already pointed out, for the symmetric diffraction conditions corresponding to the (002) and the (004) peaks, $\omega$ scans can be considered as $Q_{x}$ scans. The first point has an important consequence: in an $\omega$ scan, i.e. a $Q_{x}$ scan, all the peaks, the Bragg peak and the satellites, have the same $Q_{x}$ profile, $B\left(q_{x}\right)$, which corresponds to the cross section of the instrumental resolution function in the $\omega$ direction. This unity of the profile is of major importance when determining the observed intensity of the peaks by leastsquare fits, $O_{p}$. The 'theoretical' profile to be fitted can be written $\sum_{p=-P}^{P} b+O_{|p|} B\left[\left(Q_{x}-Q_{00 l}-p q_{\mathrm{a}}\right) / \Delta q_{x}\right]$ where $\Delta q_{x}$ is the profile width. The number of parameters to be fitted for $2 P$ satellites is therefore $P+5$ : the $P+1$ peak intensities, $b$, $Q_{00 l}, q_{\mathrm{a}}$ and $\Delta Q_{x}$. This important constraint allows one to determine very weak satellite intensities even when they are poorly separated from each other [for instance, for the (004) scan, Fig. 5]. Good fits can be obtained with a Gaussian for $B$. We find $\lambda_{\mathrm{a}}=5.0 \pm 0.2 \mu \mathrm{m}$ around the (002) Bragg peak and $\lambda_{\mathrm{a}}=5.2 \pm 0.2 \mu \mathrm{m}$ around the (004) Bragg peak.

It is of major importance to examine the consequences of the convolution in the $Q_{z}$ direction, i.e. the $\omega-2 \theta$ direction, on the effective intensities. As pointed out before, the natural width in the $q_{z}$ direction rapidly varies from peak to peak while the resolution function remains constant. Therefore, the result of the convolution leads to geometrical correction factors, GCF $=I_{\text {eff }} / I_{\text {nat }}$, which depend on the satellite order. These factors are similar to the corrections for surface X-ray diffraction measurements (Vlieg, 1997; Robach et al., 2000). In the literature devoted to the X-ray diffraction study of acoustic waves propagating on GaAs (Sauer et al., 1999), $\mathrm{LiNbO}_{3}$ (Tucoulou et al., 2001), Si (Tucoulou et al., 2000) or $\mathrm{La}_{3} \mathrm{Ga}_{5} \mathrm{SiO}_{14}$ (Roshchupkin, Irzhak, Snigirev et al., 2011), we have not found any example of calculation of such geometrical correction factors. Since the $q_{z}$ profile of each peak depends on $U$, the convolution and the calculation of the geometrical correction factors must be performed for each value of $U$. An example of such a convolution is given in Fig. 4(b). In order to calculate the geometrical correction factors, we have measured the instrumental resolution function without SAWs, assuming that the profiles of the (002) and (004) Bragg peaks are controlled by the instrumental resolution function. For the sake of simplicity, we assumed that these profiles are Gaussian,

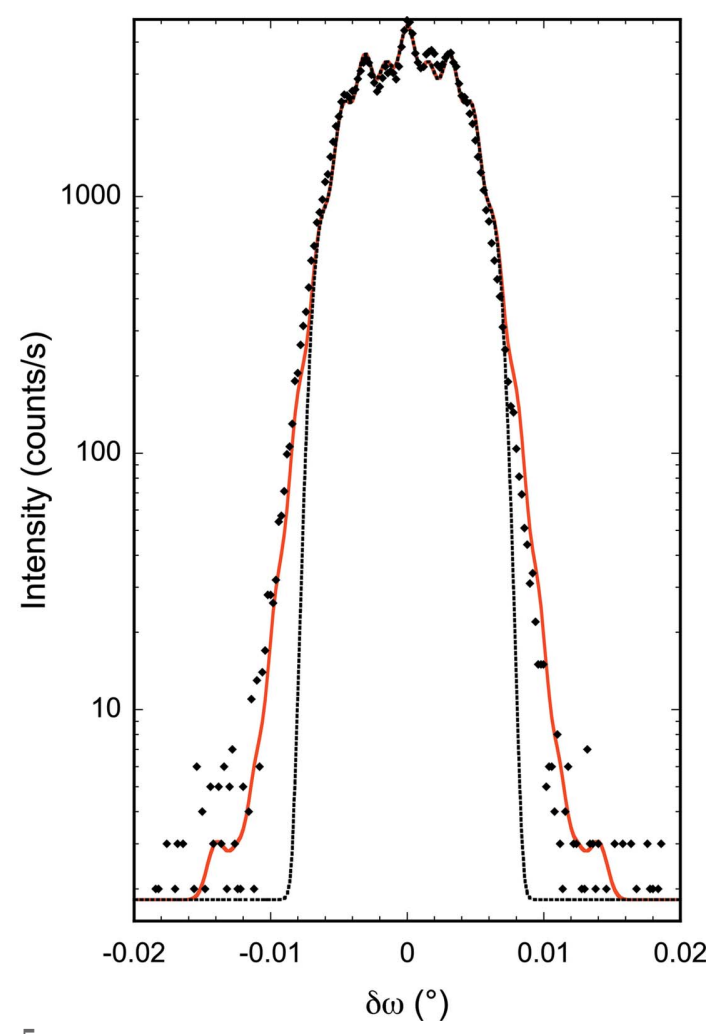

Figure 5

Fit of the rocking curve around the (004) Bragg peak for an RF power of $P_{\text {elec }}=160 \mathrm{~mW}(x=2.1 \mathrm{~mm})$. Black diamonds: observed intensity; red line: best fit with $2 \times 8$ Gaussian satellites and one central Gaussian; black dotted line: best fit with only $2 \times 4$ satellites and one central Gaussian. 
Table 1

Values of GCF, the geometrical correction factor, for different satellite orders around the (002) Bragg peak as a function of $U$.

\begin{tabular}{llllll}
\hline$U(\mathrm{pm})$ & $\mathrm{GCF}_{0}$ & $\mathrm{GCF}_{1}$ & $\mathrm{GCF}_{2}$ & $\mathrm{GCF}_{3}$ & $\mathrm{GCF}_{4}$ \\
\hline 23 & 0.142 & 0.639 & 0.708 & 0.767 & 0.805 \\
34 & 0.139 & 0.635 & 0.704 & 0.766 & 0.804 \\
62 & 0.135 & 0.621 & 0.690 & 0.758 & 0.799 \\
86 & 0.146 & 0.614 & 0.671 & 0.746 & 0.792 \\
\hline
\end{tabular}

$B\left(q_{x}, q_{z}\right) \propto \exp \left\{-\left[\left(q_{x} / \Delta q_{x}\right)^{2}+\left(q_{z} / \Delta q_{z}\right)^{2}\right]\right\} . \quad$ We $\quad$ find $\Delta q_{x}(002)=4.1 \times 10^{-4} \mathrm{~nm}^{-1}, \Delta q_{z}(002)=15 \times 10^{-4} \mathrm{~nm}^{-1}$, $\Delta q_{x}(004)=6.7 \times 10^{-4} \mathrm{~nm}^{-1}$ and $\Delta q_{z}(004)=13 \times 10^{-4} \mathrm{~nm}^{-1}$. We then performed numerically the convolution with the natural intensity by fast Fourier transform for several values of $U$ with a $1 \mathrm{pm}$ step. Table 1 illustrates the dependency of the GCF on the peak order and the SAW amplitude. It is of major importance to note that the geometrical correction factors vary significantly with the peak order - half an order of magnitude when going from the Bragg peak to the satellites and $25 \%$ when going from first order to fourth order.

For each rocking curve and each value of the incoming RF power, the observed peak intensities, $O_{p}$, are determined by least-squares fit. Then, a second least-squares fit between these measured intensities, $O_{p}$, and the calculated effective intensities taking into account the geometrical correction factors, $I_{\text {eff }-p}$, allows the determination of $U$. Table 2 illustrates the result of this double-fit procedure around the (002) Bragg peak for different incoming powers.

When performing such a procedure, a difficulty occurs for the (004) rocking curves: the kinematic approach we have chosen is not able to account for the intensity of the (004) Bragg peak while it accounts for the intensity of the satellites. This is due to the quite low value of $\tilde{\mu}_{004}$. Therefore, we exclude the (004) Bragg peak from our analysis using solely

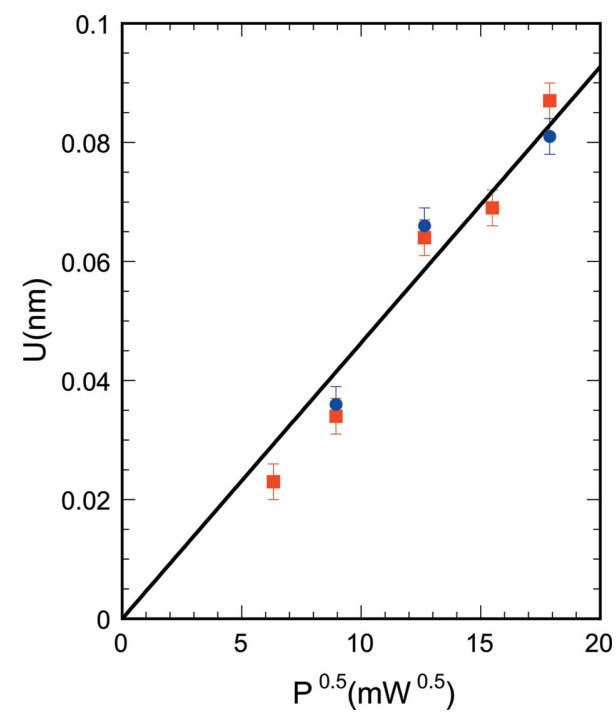

Figure 6

Measured Rayleigh mode amplitude as a function of the square root of the incoming RF power. Red squares: (002) Bragg peak; blue dots: (004) Bragg peak; black line: best fit by a proportional law $U=\gamma P^{0.5}$ leading to $\gamma=4.6 \mathrm{pm} \mathrm{mW}^{-0.5}$. the different satellite intensities. Note that previous authors have faced the same problem (Tucoulou et al., 2001). This exclusion of the Bragg peak is compensated for by the high number of satellites measurable around the (004) peak.

\section{Data analysis}

Fig. 6 shows the results obtained for $U$ from rocking curves around the (002) and (004) Bragg peaks as a function of the square root of the electric power. Two features appear clearly on this figure: first, the analyses around the (002) and (004) Bragg peaks lead to equivalent determinations of $U$; second, the $U$ values are proportional to the square root of the incoming RF power, as expected from the expressions of the acoustic Poynting vector (Royer \& Dieulesaint, 2001). For the maximum $\mathrm{CW}$ power used $(320 \mathrm{~mW})$, the parameter $U=$ $0.083 \mathrm{~nm}$ implies surface $x$ and $z$ displacements of $u_{x}(z=0)=$ $0.08 \mathrm{~nm}$ and $u_{z}(z=0)=0.11 \mathrm{~nm}$. Those correspond to surface strains $\varepsilon_{x x}=9.9 \times 10^{-5}, \varepsilon_{x z}=0, \varepsilon_{z z}=4.5 \times 10^{-5}$ (absolute values). Moreover, the acoustic power is then $2.44 \mathrm{~W} \mathrm{~m}^{-1}$ and the electromechanical conversion efficiency of the $\mathrm{IDT}_{\mathrm{e}}$ is $-18 \mathrm{~dB}$. These are good values for SAWs excited directly on $\mathrm{GaAs}$, without the addition of a traditionally more efficient piezoelectric material such as $\mathrm{LiNbO}_{3}$ or $\mathrm{ZnO}$.

This validation of our data reduction procedure allows laboratory studies of two physical questions:

(a) Using X-rays the absolute attenuation of the Rayleigh mode with distance from the IDT $_{\mathrm{e}}$ can be determined. Figs. 7

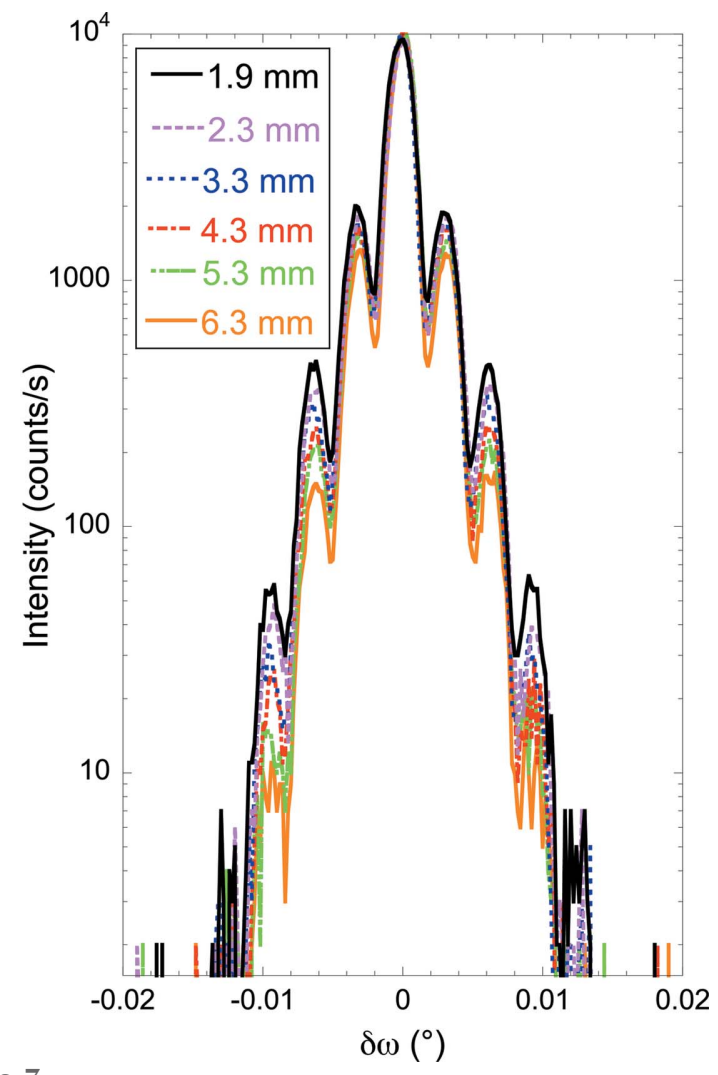

Figure 7

Rocking curves around the (002) Bragg peak for increasing distance to the $\operatorname{IDT}_{\mathrm{e}}\left(P_{\text {elec }}=160 \mathrm{~mW}\right)$. 
Table 2

Comparison between measured peak effective intensities, $O_{p}$, and best calculated peak effective intensities, $I_{\text {eff }-p}$, around the (002) Bragg peak for various incoming powers.

\begin{tabular}{lllllllllll}
\hline$P(\mathrm{~mW})$ & $O_{0}$ & $I_{\text {eff-0 }}$ & $O_{1}$ & $I_{\text {eff-1 }}$ & $O_{2}$ & $I_{\text {eff-2 }}$ & $O_{3}$ & $I_{\text {eff-3 }}$ & $O_{4}$ & $I_{\text {eff-4 }}$ \\
\hline 40 & 1 & 1 & 0.034 & 0.035 & 0.0011 & 0.00086 & & & & \\
80 & 1 & 1 & 0.074 & 0.074 & 0.0044 & 0.0041 & 0.00062 & 0.00011 & & \\
160 & 1 & 1 & 0.19 & 0.19 & 0.044 & 0.040 & 0.0056 & 0.0039 & 0.00075 & 0.00022 \\
240 & 1 & 1 & 0.20 & 0.21 & 0.056 & 0.052 & 0.0089 & 0.0061 & 0.0012 & 0.00040 \\
320 & 1 & 1 & 0.22 & 0.22 & 0.10 & 0.10 & 0.025 & 0.022 & 0.00039 & 0.0026 \\
\hline
\end{tabular}

and 8 illustrate this study. For distances larger than $x=2 \mathrm{~mm}$ from the $\mathrm{IDT}_{\mathrm{e}}$, we find the Rayleigh mode to be exponentially damped with an attenuation coefficient of $5.9 \mathrm{~dB} \mathrm{~cm}^{-1}$ at $550 \mathrm{MHz}$. This value is consistent with existing data: Slobodnik reported about $15 \mathrm{~dB} \mathrm{~cm}^{-1}$ at $1 \mathrm{GHz}$ for different cut and propagation directions (Slobodnik, 1972). From this value, we derive $4.5 \mathrm{~dB} \mathrm{~cm}^{-1}$ at $550 \mathrm{MHz}$, assuming a squared frequency variation of the attenuation [which is valid when the wave interacts with the thermal phonons (Truell et al., 1969)]. This estimation is satisfyingly close to our measured value. We also find the SAW amplitude to be identical on either side of the $\mathrm{IDT}_{\mathrm{e}}$, proving that no spurious reflections off the receiving IDT lead to destructive interferences of the wave amplitude.

(b) The important relative variations of the satellite peaks with the Rayleigh mode amplitude allow one to study it as a function of Mod SAW duty cycles, when modulating the RF power, as illustrated in Fig. 9. We obtained rocking curves around the (002) Bragg peak for several duty cycles of constant period, $T_{\mathrm{mod}}=20 \mu \mathrm{s}$, and of variable 'on' time, $T_{\mathrm{on}}$. This implies that, whereas the average displacement should decrease for weaker duty cycles, the instantaneous displacement seen when the SAW is 'on' should be identical. As shown in the first two columns of Table 3, for each duty cycle labelled by the ratio $\tau=T_{\text {on }} / T_{\text {mod }}$, we can satisfactorily fit the rocking curve by a linear combination of the rocking curves obtained

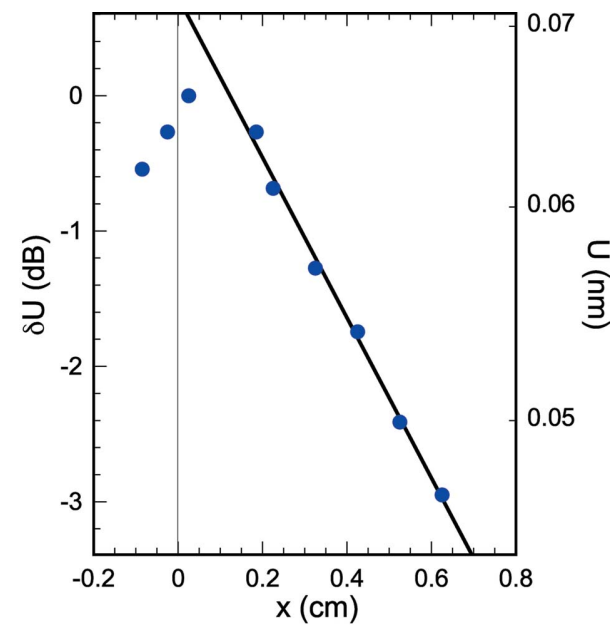

Figure 8

Variation of the Rayleigh mode amplitude as a function of the distance to the $\operatorname{IDT}_{\mathrm{e}}\left(P_{\text {elec }}=160 \mathrm{~mW}\right)$. Blue dots: variation of the measured acoustic amplitude in $\mathrm{dB}$ (left axis) and its corresponding $U$ value in $\mathrm{nm}$ (right axis in log scale); black line: exponential fit. The vertical black line at $x=0$ corresponds to the right edge of the $\mathrm{IDT}_{\mathrm{e}}$. for the two extreme conditions, $\tau=0$ and $\tau=1$. Fig. 10 illustrates the quality of the fit obtained with such a linear combination. This result paves the way to a determination of the acoustic amplitude using non-continuous wave excitation. For this determination, the Bragg peak must be excluded since its intensity depends on the ratio $\tau$, but the relative satellite intensities are the same for any $\tau$ since they are only due to the times during which the incoming power is 'on'. The third column of Table 3 gives the acoustic wave amplitude, $U$, measured using the first three satellites around the (002) Bragg peak. We verified, using the CW SAW rocking curve, that the determination of $U$ using the three satellites plus the Bragg peak leads to the same result as the determination with only the three satellites. This possibility of determining $U$ under Mod SAW is important since it allows the use of high electric power, which for a continuous wave excitation would damage the transducers.

\section{Discussion and conclusion}

While a strict justification of our kinematic approach would imply a dynamic calculation, the overall results we obtained good agreement between the values of $U$ determined around

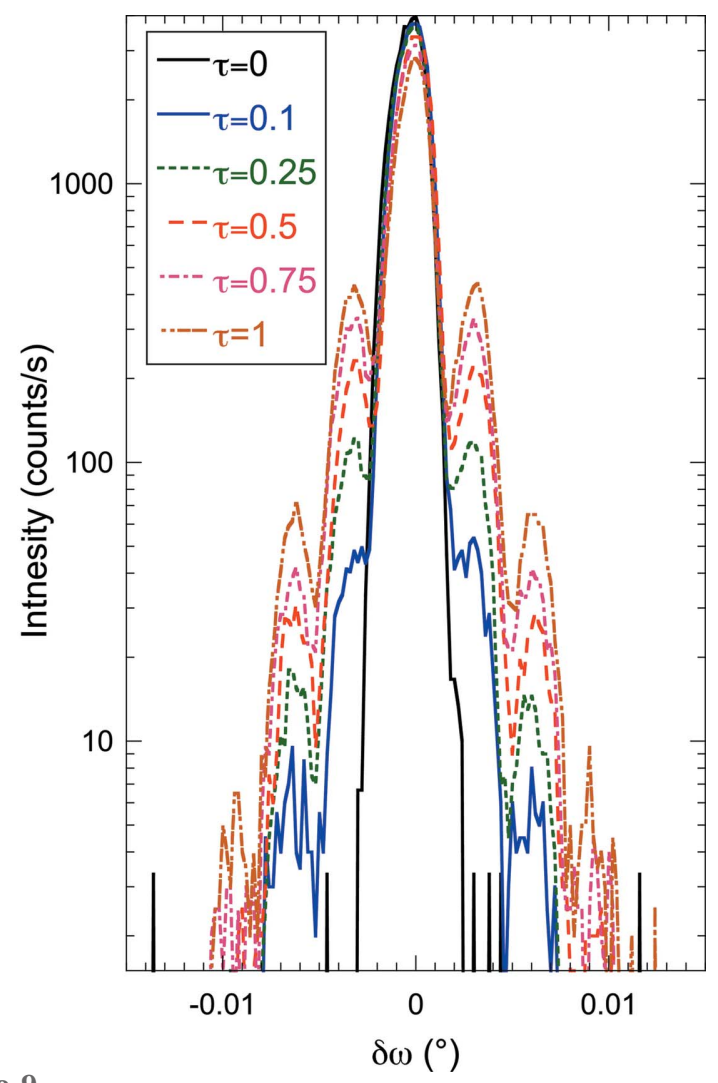

Figure 9

Rocking curves around the (002) Bragg peak for duty cycles of constant period, $T_{\mathrm{mod}}=20 \mu \mathrm{s}$, and of variable 'on' time, $T_{\mathrm{on}}=\tau T_{\bmod }(x=2.1 \mathrm{~mm}$, $\left.P_{\text {elec }}=160 \mathrm{~mW}\right)$. 
Table 3

Determination of $U$ using duty cycles.

For each duty cycle, $\alpha$ corresponds to a fit by a linear combination of the $100 \%$ and $0 \%$ rocking curves, $(1-\alpha) I(\tau=0)+\alpha I(\tau=1)$. For the $100 \%$ duty cycle the value of $U$ determined using the Bragg peak and the satellites is marked by an asterisk.

\begin{tabular}{lll}
\hline$\tau=T_{\text {on }} / T_{\text {tot }}$ & $\alpha$ & $U(\mathrm{~nm})$ \\
\hline 0.1 & $0.08 \pm 0.07$ & $0.057 \pm 0.003$ \\
0.25 & $0.33 \pm 0.07$ & $0.051 \pm 0.003$ \\
0.5 & $0.58 \pm 0.07$ & $0.051 \pm 0.003$ \\
0.75 & $0.79 \pm 0.07$ & $0.050 \pm 0.003$ \\
1 & & $0.054 \pm 0.003\left(0.053^{*}\right)$ \\
\hline
\end{tabular}

the (002) and the (004) Bragg peaks, exponential decay of $U$ with distance from the $\mathrm{IDT}_{\mathrm{e}}$, determination of a constant $U$ for variable duty cycle - validate our kinematic approach. This approach is based on four main points: (i) good resolution in the $q_{x}$ direction, $\Delta q_{x} \simeq 5 \times 10^{-4} \mathrm{~nm}^{-1}$, obtained using a fourcrystal monochromator and a two-crystal analyser; (ii) reduction of the footprint to the crystal region affected by the acoustic wave using appropriate masks; (iii) calculation of the Rayleigh mode in the framework of linear elasticity of continuous media; (iv) taking into account 'geometrical'

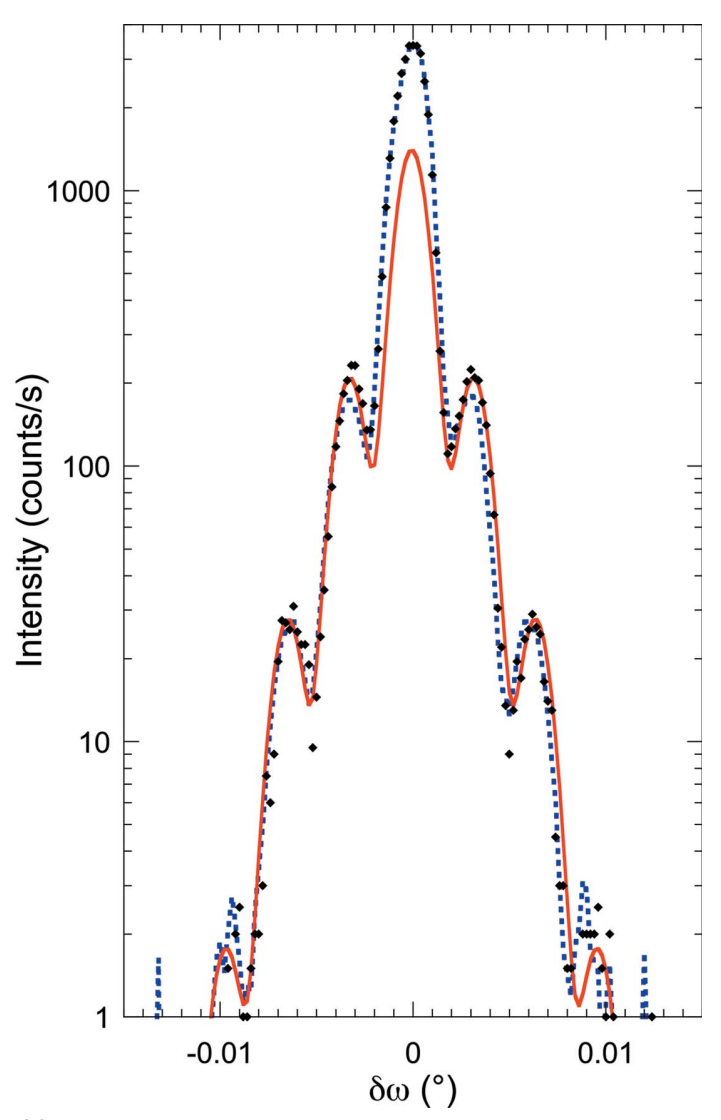

Figure 10

Rocking curve around the (002) Bragg peak for a duty cycle of $50 \%(x=$ $2.1 \mathrm{~mm}, P_{\text {elec }}=160 \mathrm{~mW}$ ). Black diamonds: observed intensity; blue dotted line: best linear combination of 0 and $100 \%$ rocking curves; red full line: best calculated curve with a determination of $U$ using only the three satellites. (Note the discrepancy between the observed and the calculated curves near the Bragg peak.) corrections due to finite instrumental resolution in the $q_{z}$ direction, $\Delta q_{z} \simeq 10^{-3} \mathrm{~nm}^{-1}$.

It is worth noting that the determination of $U$ implies measurements of quite low satellite intensities in the immediate vicinity of the Bragg peak, for example $I_{3} / I_{0}=10^{-3}$ for $U=0.05 \mathrm{~nm}$ around (002) and $I_{6} / I_{0}=2 \times 10^{-4}$ for $U=0.036 \mathrm{~nm}$ around (004). These low values hinder a strict statistical estimate of the errors. Nevertheless, a study of the least-squares dependency on $U$ allows one to estimate the uncertainty $\Delta U / U$ to be of the order of 0.06 . The exponential damping of the SAW leads to a relative variation of \pm 0.07 for $U$ in the $2 \mathrm{~mm}$ width of the $\mathrm{X}$-ray spot around the (002) Bragg peak. This variation is quite similar to our statistical uncertainty and allows us to neglect the SAW attenuation along its propagation direction in the data modelling that we have performed. Lastly, the measure of $U$ depends on the correctness of the expression chosen for the acoustic mode. A minute error on the IDT orientation with respect to $\langle 110\rangle$ will lead to a modification of expression (1), the acoustic wave no longer being a Rayleigh mode but a pseudo Rayleigh mode. A quantitative analysis of such an effect is far beyond the scope of this paper.

To conclude, we show that using both a careful experimental procedure and an accurate data analysis including instrumental convolution, measurements of surface acoustic wave amplitude can be performed on a laboratory X-ray diffractometer, provided the SAW spatial frequency is such that $q_{\mathrm{a}}>\Delta q_{x} / 2$. This work was performed on a GaAs monocrystal. However, since the satellite features appear in the in-plane direction, this diffraction technique can be applied to determine acoustic amplitudes in thin strained films. The ability to perform such determinations with a laboratory diffractometer is important given the versatility of experimental studies using surface acoustic waves.

\section{Acknowledgements}

We thank Christophe Rafaillac and Chris Lelong for help with the sample holder adapted to the RF cables, Loïc Beccera for help with the IDTs, and Jean-Yves Prieur for fruitful discussions and advice. This work was supported by the French Agence Nationale de la Recherche (ANR13-JS04-0001-01).

\section{References}

Bojahr, A., Herzog, M., Schick, D., Vrejoiu, I. \& Bargheer, M. (2012). Phys. Rev. B, 86, 144306.

Cottam, R. I. \& Saunders, G. A. (1973). J. Phys. C Solid State Phys. 6, 2105-2118.

Entin, I. R., Khrupa, V. I. \& Datsenko, L. I. (1990). J. Appl. Cryst. 23, 355-358.

Hauer, R. \& Burns, D. J. (1975). Appl. Phys. Lett. 27, 524.

Hermelin, S., Takada, S., Yamamoto, M., Tarucha, S., Wieck, A. D., Saminadayar, L., Bäuerle, C. \& Meunier, T. (2011). Nature, 477, 435-438.

Insepov, Z., Emelin, E., Kononenko, O., Roshchupkin, D. V., Tnyshtykbayev, K. B. \& Baigarin, K. A. (2015). Appl. Phys. Lett. 106, 023505.

Lean, E. G. H. \& Powell, C. G. (1972). Proc. IEEE, 93, 1939. 
Li, W., Buford, B., Jander, A. \& Dhagat, P. (2014). Phys. B Condens. Matter, 448, 151-154.

Lima, M. M. de, Alsina, F., Seidel, W. \& Santos, P. V. (2003). J. Appl. Phys. 94, 7848.

Robach, O., Garreau, Y., Aïd, K. \& Véron-Jolliot, M. B. (2000). J. Appl. Cryst. 33, 1006-1018.

Roshchupkin, D. V. \& Brunel, M. (1993). Rev. Sci. Instrum. 64, 379.

Roshchupkin, D. V., Irzhak, D. V., Plotitsyna, O. A., Fakhrtdinov, R. R., Buzanov, O. A. \& Sergeev, A. P. (2011). J. Surf. Invest. 6, 490.

Roshchupkin, D. V., Irzhak, D., Snigirev, A., Snigireva, I., Ortega, L. \& Sergeev, A. (2011). J. Appl. Phys. 110, 124902.

Royer, D. \& Dieulesaint, E. (2001). Elastic Waves in Solids. Heidelberg: Springer-Verlag.

Sanada, H., Sogawa, T., Gotoh, H., Onomitsu, K., Kohda, M., Nitta, J. \& Santos, P. V. (2011). Phys. Rev. Lett. 106, 216602.

Sauer, W., Streibl, M., Metzger, T. H., Haubrich, A. G. C., Manus, S., Wixforth, A. \& Peisl, J. (1999). Appl. Phys. Lett. 75, 639.

Schelokov, I. A., Roshchupkin, D. V., Irzhak, D. V. \& Tucoulou, R. (2004). J. Appl. Cryst. 37, 52-61.

Schülein, F. J. R., Zallo, E., Atkinson, P., Schmidt, O. G., Trotta, R., Rastelli, A., Wixforth, A. \& Krenner, H. J. (2015). Nat. Nanotechnol. 10, 512-516.
Singh, U. \& Adenwalla, S. (2015). Nanotechnology, 26, 255707.

Slobodnik, A. J. (1972). Electron. Lett. 8, 307.

Thevenard, L., Camara, I. S., Majrab, S., Bernard, M., Rovillain, P., Lemaître, A., Gourdon, C. \& Duquesne, J.-Y. (2016). Phys. Rev. B, 93, 134430 .

Truell, R., Elbaum, C. \& Chick, B. B. (1969). Ultrasonic Methods in Solid State Physics. New York, London: Academic Press.

Tucoulou, R., de Bergevin, F., Mathon, O. \& Roshchupkin, D. (2001). Phys. Rev. B, 64, 134108.

Tucoulou, R., Mathon, O., Ferrero, C., Mocella, V., Roshchupkin, D. V. \& Kumon, R. E. (2005). J. Appl. Phys. 97, 113505.

Tucoulou, R., Pascal, R., Brunel, M., Mathon, O., Roshchupkin, D. V., Schelokov, I. A., Cattan, E. \& Remiens, D. (2000). J. Appl. Cryst. 33, 1019-1022.

Tucoulou, R., Roshchupkin, D. V., Mathon, O., Schelokov, I. A., Brunel, M., Ziegler, E. \& Morawe, C. (1998). J. Synchrotron Rad. 5, 1357-1362.

Tucoulou, R., Roshchupkin, D. V., Schelokov, I. A., Brunel, M., Ortega, L., Ziegler, E., Lingham, M., Mouget, C. \& Douillet, S. (1997). Nucl. Instrum. Methods Phys. Res. Sect. B, 132, 207-213.

Vlieg, E. (1997). J. Appl. Cryst. 30, 532-543.

Zolotoyabko, E. \& Polikarpov, I. (1998). J. Appl. Cryst. 31, 60-66. 